\title{
A Letter From the Guest Editor
}

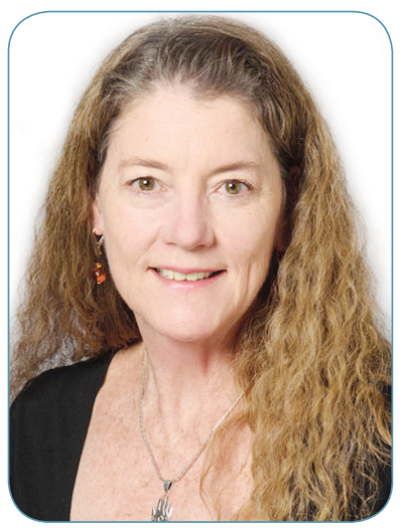

look forward to attending the American Society of Hematology (ASH) annual meeting every year. The 2018 meeting was filled with excitement generated by scientific innovation and improved understanding of the complexity of managing patients with hematologic malignancies and blood disorders. The challenge for me, as for other advanced practitioners and hematology/oncology professionals, is how to rapidly and effectively integrate this science into the day-to-day management of our patients with cancer (Figure 1).

Patient-centric care that integrates both patient- and disease-specific attributes is critical to tailoring treatment to optimize outcomes. Most of these principles apply across both liquid and solid tumors. Equally important are the numerous studies presented focused on patient-reported outcomes, value-based care models, and the integration of health technology relative to programmatic outcomes and professional development. The almost frenetic pace of scientific innovation, and the continued shift toward value-based care and associated regulatory mandates, require constant engagement with reliable sources of information.

\section{SEEKING OUT INFORMATION}

So what is an advanced practitioner to do? We are accustomed to attending meetings, such as $\mathrm{ASH}$, the annual meeting of the American Society of Clinical Oncology (ASCO), or JADPRO Live, to hear from thought-leaders and colleagues about some of these advances. But we have to seek out other resources as well. Most presentations at major meet-

J Adv Pract Oncol 2019;10(suppl 2):2-3

https://doi.org/10.6004/jadpro.2019.10.2.15 ings, such as ASH, are presented in abstract form and have not yet been published in peer-reviewed journals. Yet these educational resources and others can help advanced practitioners stay on top of the quickly changing information that is characteristic of the world of treating patients with cancer.

Planning your schedule for what to go to at ASH is almost impossible. Even with 12- to 14-hour days, there is no way to attend all of the sessions of interest. As the guest editor for this supplement to JADPRO, I was tasked with choosing nine abstracts from ASH 2018 that would be of particular interest to the journal's advanced practitioner readers. With all the ground-breaking science presented at the meeting, how could I possibly just choose nine?

The principles that guide my practice are grounded in applying scientific innovations and programmatic concepts to individualize treatment, maximize each treatment option, prevent or mitigate treatment-emergent adverse events, and improve or maintain patient quality of life in a way that is sustainable for both the patient and the practice. You'll see an illustration of this concept in Figure 1.

With this paradigm in mind, I chose abstracts I thought represented a variety of hematologic malignancies and offered insight into the following:

1. New classes of drugs, novel pathways and targets, new administration techniques, and unique adverse event profiles

2. Long-term treatment outcomes for patients with incurable cancers

3. Emerging scientific principles for risk stratification, monitoring, and management of hematologic malignances over time (newly diagnosed or relapsed/refractory)

4. Topics chosen as late-breaking or plenary sessions

\section{IMPLICATIONS FOR THE AP}

Once I chose the nine key abstracts, I invited two of my colleagues-Josh Epworth, ARNP, from the University of Washington, and Lindsey M. Lyle, MS, PA-C, from the University of Colorado, An- 


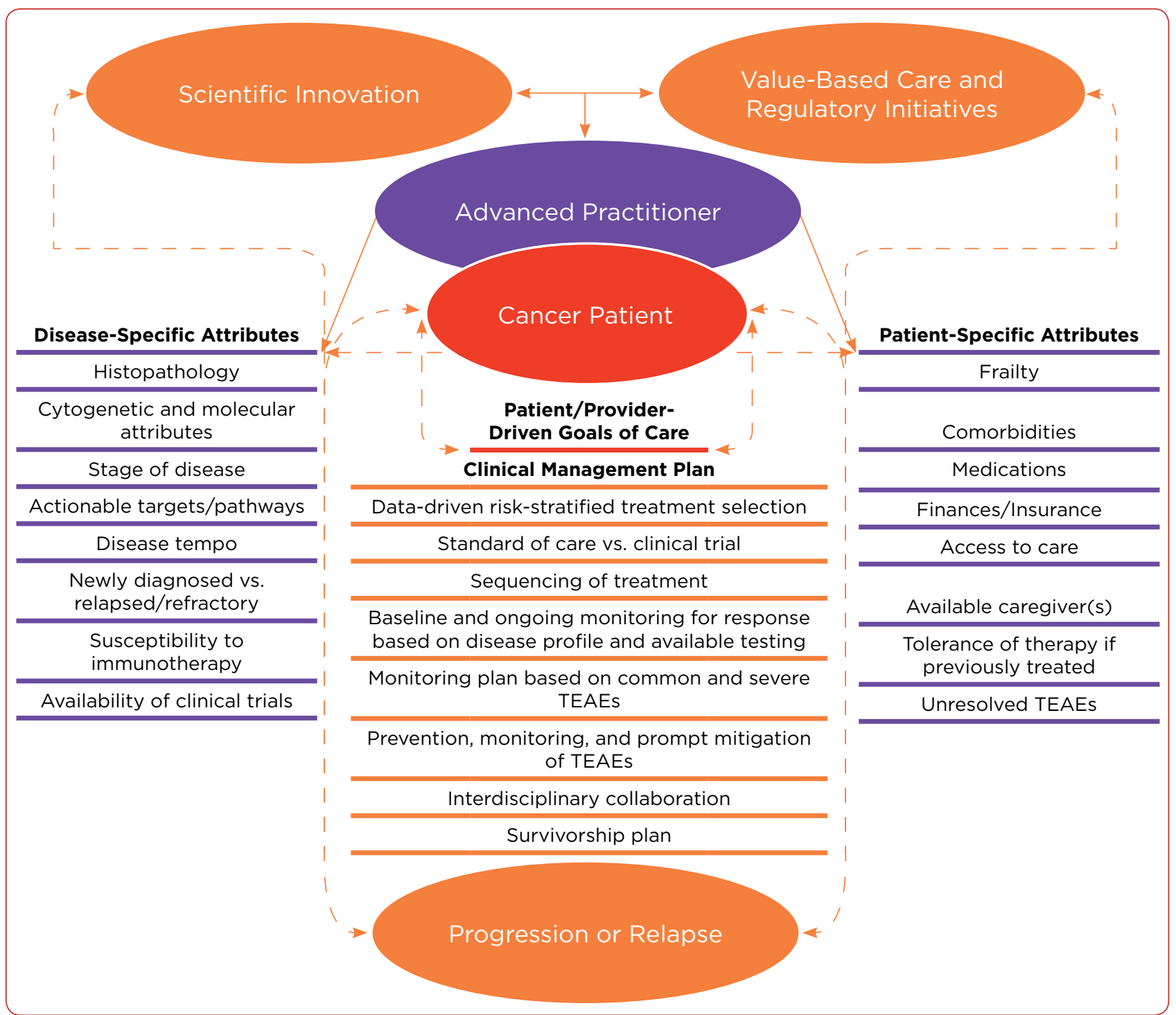

Figure 1. Integrating scientific innovation, value-based care, and regulatory initiatives into the tailored treatment of patients with cancer. TEAE = treatment-emergent adverse event.

schutz Medical Campus-to join me in providing some commentary to highlight what's particularly relevant for the advanced practitioner. As a head start, back in December we filmed some short videos on site at ASH, seeking to provide some quick narratives to give viewers a taste of what was going on at the meeting. You can view the videos at advancedpractitioner.com/highlights.

In the pages of this follow-up supplement to JADPRO, Josh, Lindsey, and I have each taken three abstracts and expanded upon those video commentaries to bring you a more in-depth look at each abstract and what it might mean to your practice, either today or in the future. In each of our sections, you'll first see a reproduction of the three abstracts for your review and reference, followed by our commentaries.

Learning to critically review abstracts to inform your practice requires vigilance and perseverance. On behalf of JADPRO, Josh, Lindsey, and I hope to offer you insight into how we critically review abstracts to extract findings relevant to our practice, so you can do the same when you attend meetings in the future. We hope you find this to be a valuable reference for your practice.

- Sandra E. Kurtin, PhD, ANP-C, AOCN ${ }^{\circledR}$ The University of Arizona Cancer Center Guest Editor 